\title{
LOW-FREQUENCY GYROTRONS FOR FUSION STUDIES
}

\author{
V. E. Zapevalov, ${ }^{1 *}$ Yu. K. Kalynov, ${ }^{1}$ V.K. Lygin, ${ }^{1 \dagger}$ \\ O. V. Malygin, ${ }^{1}$ S. A. Malygin, ${ }^{3}$ M. A. Moiseev,${ }^{1}$ \\ V. N. Manuilov, ${ }^{2}$ E. A. Soluyanova, ${ }^{3}$ E. M. Tai, ${ }^{3}$ and \\ V. I. Khizhnjak ${ }^{1}$
}

UDC 621.385.69.01

\begin{abstract}
A number of experimental plasma devices require high-power sources of electromagnetic radiation in a range of 5 to $30 \mathrm{GHz}$. In this paper, we consider gyrotrons as such sources. The difficulties of creating long-wavelength gyrotrons are discussed and the possibility to overcome them is shown for already existing and currently developed oscillators. We present the results of studies aimed at development of high-power, long-pulse, low-frequency gyrotrons in Russia, as well as the designs and performance parameters of industrial gyrotrons operated at frequencies 5 and $28 \mathrm{GHz}$ with an output power of $0.5 \mathrm{MW}$. Several design versions of the gyrotrons with operating frequencies 17.5 and $28 \mathrm{GHz}$, an output power of up to $1 \mathrm{MW}$, and a pulse duration of up to 5 s are discussed and the design features which make it possible to overcome the difficulties of their manufacturing are considered.
\end{abstract}

\section{INTRODUCTION}

It is well known that the highest-power (with output-radiation power about or more than $1 \mathrm{MW}$ ) and longest-pulse (with pulse duration from several seconds up to the continuous regime) gyrotrons are used successfully in the experiments on electron cyclotron heating of the plasma in tokamaks and stellarators $[1,2]$. Therefore, until recently, the main efforts have been aimed at increasing the energy of the output radiation (power and pulse duration) of the gyrotrons operated in the short-wavelength part of the millimeter-wave range. The main practical application of low-frequency gyrotrons (operated in the short-wavelength part of the centimeter-wave range) has been their use in new technological processes [3]. Even in the long-term outlook, the required output power of such gyrotrons did not exceed several tens of kilowatts. However, recently, interest in long-pulse, low-frequency megawatt gyrotrons has significantly increased. In particular, this is caused by the advance in the works on plasma heating by using Bernstein waves in spherical tokamaks $[4,5]$. Such experiments require sources of the electromagnetic radiation which are operated in a range of 15 to $30 \mathrm{GHz}$ and generate an output power of the order of $1 \mathrm{MW}$ in pulses up to $5 \mathrm{~s}$ long. In what follows we consider gyrotrons as such radiation sources.

The above-mentioned comparatively low-frequency gyrotrons have their own specific problems, features of individual components, and certain design challenges. Table 1 shows the main differences in the problems encountered when developing high-power, long-pulse gyrotrons of the millimeter- and centimeterwave ranges.

\footnotetext{
* zapev@appl.sci-nnov.ru

$\dagger$ Deceased.
}

${ }^{1}$ Institute of Applied Physics of the Russian Academy of Sciences; ${ }^{2}$ N. I. Lobachevsky State University of Nizhny Novgorod ; ${ }^{3}$ GYCOM Ltd., Nizhny Novgorod, Russia. Translated from Izvestiya Vysshikh Uchebnykh Zavedenii, Radiofizika, Vol.49, No.3, pp. 207-218, March 2006. Original article submitted July 25, 2005; accepted September 27, 2005.

$$
\text { 0033-8443/06/4903-0185 (C) } 2006 \text { Springer Science+Business Media, Inc. }
$$


TABLE 1.

\begin{tabular}{|c|c|c|}
\hline problems $^{*}$ & millimeter range & centimeter range \\
\hline mode selection & + & - \\
\hline heat load in the cavity & + & - \\
\hline output converter & + & ++ \\
\hline electron beam & $\begin{array}{c}+ \\
\text { initial-velocity } \\
\text { spread }\end{array}$ & $\begin{array}{c}+ \\
\text { nonadiabatic } \\
\text { effects }\end{array}$ \\
\hline collector & + & ++ \\
\hline output window & + & - \\
\hline
\end{tabular}

* "-" denotes the absence of problems, "+", the presence of problems, and "++", the presence of difficult problems. Estimations show that in the considered frequency range, operation at the modes corresponding to the root of order $\nu \approx 10$ of the derivative of a Bessel function of the first kind is sufficient to provide an acceptable level of the specific thermal load in the cavity at the megawatt level of the output power up to the continuous regime for the cavity radius $R_{\text {cav }} \approx 1.5 \lambda$, where $\lambda$ is the operating wavelength.

For long-wavelength gyrotrons, the problem of mode competition is not as acute as in the case of short-wavelength tubes. This is related to the fact that even when the electrodynamic system works with low types of modes, its size still remains rather large to ensure that the specific thermal load in it is admissible for a high output power and a long pulse duration.

Selection of a suitable type of the operating mode, which provides the most efficient conversion into a linearly polarized Gaussian beam usually required in practical applications, is the most topical problem for long-wavelength gyrotrons. The apertures of the elements of a quasi-optical converter are comparatively small on the wavelength scale (otherwise the overall dimensions of a gyrotron would be prohibitively large), thus causing significant diffraction losses. Hence, in the centimeter-wave range (especially in its longwavelength part) the optimal design of the output converter of the operating mode can differ significantly from the conventional designs of the quasi-optical converters used in the gyrotrons operated in the millimeterwavelength range.

In the centimeter range, the problems related to the electron optics, i.e., the formation of a high-power helical electron beam and its consequent landing on the collector with an admissible specific thermal load are also different. In the millimeter-wavelength range, the elevation of electron trajectories over the cathode surface and their pitch in the area of the electron-beam formation are small, and the main reason for the spread of transverse electron velocities is the spread of their initial velocities due to various inhomogeneities and roughness of the emitter surface.

In the centimeter range, in which the operating regime of the electron gun is closer to the critical one and the electron trajectories fill the cathode-anode gap almost completely, the greatest contribution to the spread of transverse velocities is made by nonadiabatic effects in the region of the electron-beam formation.

In gyrotrons of the millimeter-wave range, an increase in the area of the electron-beam landing in order to reduce the specific thermal load is achieved comparatively easily by creation of additional static or dynamic magnetic fields in the collector region with the help of magnetic coils that distribute or displace magnetic-field lines along the collector. In the centimeter wave range, the problem of increasing the area of the electron-beam landing on the collector turns out to be more complicated due to the nonadiabatic behavior of electron motion in weak magnetic fields in the collector region. In this case, predicting the longitudinal distribution of the thermal load along the collector is not simple. It requires a detailed trajectory analysis for each specific case, which will be shown further.

The problem of the output window in the centimeter-wave range is not as acute as that in the millimeter-wave range. Due to a long wavelength of the output radiation, the window whose thickness is sufficient for mechanical strength can be comparatively wideband at low total losses of the output power in it. 


\section{INDUSTRIAL LOW-FREQUENCY GYROTRONS}

In late 1980s, the program for additional plasma heating in a T-10 tokamak by using the lower-hybrid wave was considered. To do this, a high-power source of electromagnetic radiation with a frequency of about $5 \mathrm{GHz}$ was required. To support this program, the Research Institute "Salyut" (Nizhny Novgorod, Russia) developed and manufactured a long-pulse gyrotron with a $5 \mathrm{GHz}$ operating frequency and a $0.5 \mathrm{MW}$ outputradiation power in 1990. Since the specific features of low-frequency gyrotrons manifest themselves in this gyrotrons most evidently and the data on this "super-long-wavelength" device were presented only briefly in [6], we will consider it in more detail.

A general view of the gyrotron is shown in Fig. 1, and its basic parameters, in Table 2. Choice of the operating mode $\mathrm{TE}_{0,1,1}$ was based on the considerations of decreasing the required volume occupied by the uniform magnetic field. A comparatively short, low-Q cavity (with the $\mathrm{Q}$-factor $Q \approx 200$ ) in the form of a section of a weakly irregular cylindrical waveguide was used. The diameter of the cylindrical section was $73.2 \mathrm{~mm}$. The reflection from the output end of the cavity was decreased due to a smooth coupling with the output waveguide. The diameter of the latter excluded excitation of the closestfrequency parasitic modes, which made it possible to use a short waveguide transition (with a length of about $3 \lambda$ ). The bend in the waveguide transition profile, which is absolutely inadmissible in millimeter-wave gyrotrons since it leads to parasitic transformation of the operating mode, does not cause any problems here due the rare mode spectrum.

When operating at the lowest modes, the optimal generation regimes are realized for comparatively low values of the electron-beam current. To increase the efficiency-optimal electron-beam current and, consequently, the power of the output radiation, the radius of the beam in the cavity was chosen to be significantly wider than $R_{\text {opt }}=17.6 \mathrm{~mm}$ required for the maximum coupling with the mode field (the average radius of the beam was $25.6 \mathrm{~mm}$ ). In the millimeter-wave range, in which one has to work at higher modes under conditions of a dense spectrum of the cavity eigenfrequencies, this way for solving the problem would be impeded by the competition of modes adjacent to the operating one. As a result of an increase in the radius of the electron beam, the optimal current was increased up to $25 \mathrm{~A}$ and the corresponding generated power, according to calculations, was higher

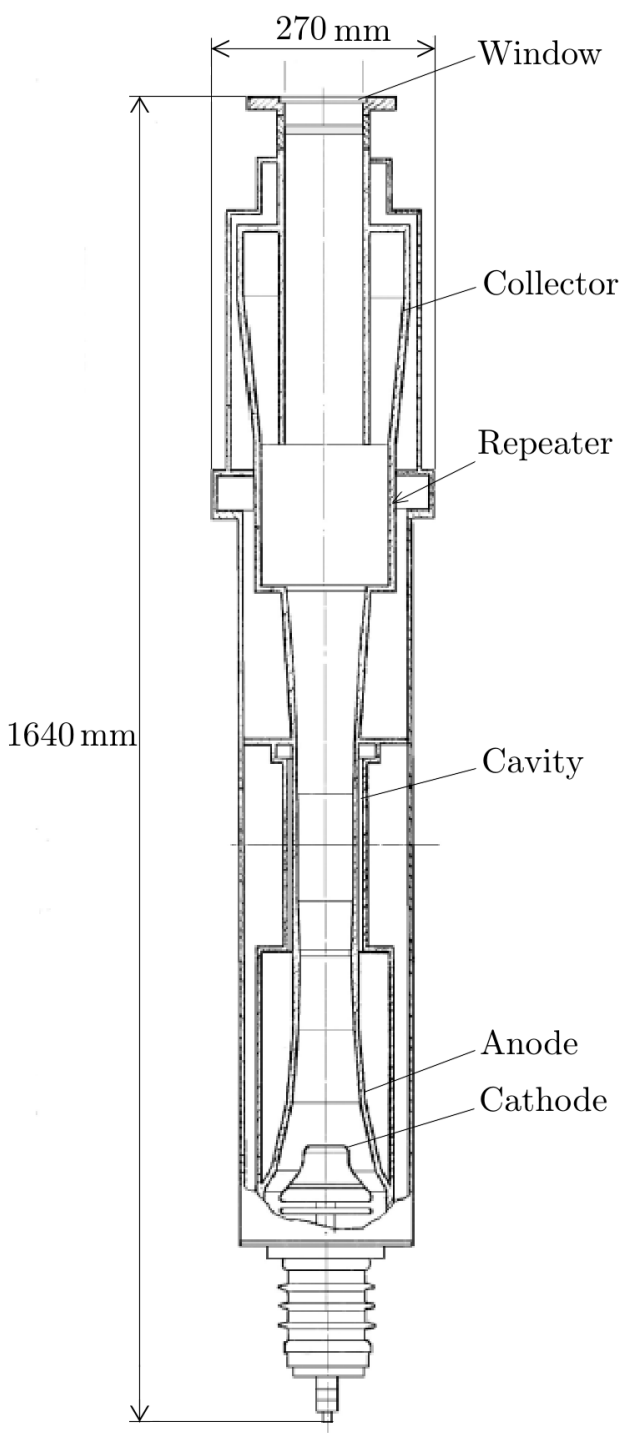

Fig. 1. General view of the gyrotron with an operating frequency of $5 \mathrm{GHz}$. than $600 \mathrm{~kW}$ for an efficiency of about $35 \%$.

To output a high-power electron beam to the collector of large diameter, while retaining the structure of the generated microwave radiation, a waveguide mode repeater, which was made as an axisymmetric shielded break of a cylindrical waveguide, was used. The tapered collector was coupled with the cylindrical shield of the repeater, and the electron beam was output to the collector through a ring slot between the shield and the output waveguide (see Fig. 1). The scheme used to separate the electromagnetic wave and the 
electron beam made it possible to make the overall dimensions of the output gyrotron module at least twice as small compared with the case of using the conventional (present-day) axially nonsymmetric quasi-optical converter.

TABLE 2.

\begin{tabular}{|c|c|}
\hline Operating mode & $\mathrm{TE}_{0,1,1}$ \\
\hline Frequency, $\mathrm{GHz}$ & 5 \\
\hline Operating voltage, $\mathrm{kV}$ & 70 \\
\hline Beam current, $\mathrm{A}$ & 25 \\
\hline Pulse duration, $\mathrm{s}$ & 1 \\
\hline Efficiency, \% & 30 \\
\hline Output power, MW & 0.5 \\
\hline
\end{tabular}

The principle of repeater operation is that the operating mode $\mathrm{TE}_{0,1}$ excites the field in the repeater space, which is the superposition of the $\mathrm{TE}_{0,1}$ and $\mathrm{TE}_{0,2}$ modes of a cylindrical waveguide. The diameter of the cylindrical shield is determined by the condition of equal power transfer from the operating mode of the cavity to the waves of the repeater. The length of the shield satisfies the condition of preservation of the initial amplitude-phase correlations when the repeater space is excited. In this case, the repeater waves excite the operating mode $\mathrm{TE}_{0,1}$ in the output waveguide with efficiency over $99 \%$.

The geometry of the magnetron injection gun was chosen with account for design limitations stipulated by the used magnetic system. To form a beam with average intracavity radius $25.6 \mathrm{~mm}$ for the limited electric field at the cathode (no greater than $5 \mathrm{kV} / \mathrm{mm}$ ), the average radius of the emitter belt was chosen equal to $R_{\text {cat }}=45 \mathrm{~mm}$ for a $4 \mathrm{~mm}$ width. Thus, for an operating current of $20-30 \mathrm{~A}$, the density of the emission current did not exceed $3 \mathrm{~A} / \mathrm{cm}^{2}$. The over-magnetization coefficient equal to the ratio of the magnetic-field intensities in the operating space of the cavity $\left(B_{\mathrm{o}} \approx 0.2 \mathrm{~T}\right)$ and at the cathode $\left(B_{\text {cat }} \approx 0.06 \mathrm{~T}\right)$ was about 3 . For an accelerating voltage of $70 \mathrm{kV}$, the gun realized a weakly supercritical regime of operation, and the beam with regularly crossing trajectories was formed. The resonant mechanism of increase in the electron-velocity spread under the space-charge effect [7] takes place in such beams. To reduce the effect of this mechanism, the configuration of the gun electrodes was optimized in an appropriate way. Fast escape of particles from the emitter was achieved by using the maximum admissible angle between the magnetic-field line and the emitter surface, which was $16^{\circ}$. To increase the oscillatory velocities of the electrons escaping from the emitter section which was closest to the resonator, the angle between the cathode surface and the tube axis at the end of the first trajectory revolution was made close to the inclination angle of the magnetic-field line. At the maximum of the trajectory elevation during the second revolution, the cathode ended sharply. As a result, the particles then moved in a sharply decreasing electric field.

Uniform landing of the high-power electron beam on the collector was achieved by means of additional coils that produced a static magnetic field guiding the particles into the ring slot between the shield and the output waveguide (see Fig. 1). It was assumed that the device was operated in the pulsed regime with the pulse duration $\tau_{\mathrm{p}} \sim 1 \mathrm{~s}$ and pulse period-to-period ratio equal to 300 . In this case, the configuration of the collector and the produced turbulent flow of the cooling liquid make it possible to dissipate up to $2.5 \mathrm{MW}$ of the idle power on the collector.

Operation of the gyrotron requires that the magnetic field produced by the solenoid has a uniform section of at least $300 \mathrm{~mm}$ in length and an inductance of about $0.2 \mathrm{~T}$. The inner diameter of the winding of such a solenoid is 200-250 mm, depending on its type and is determined by the design of the gyrotron. Thus, the required magnetic field must be produced in a comparatively large volume (about $0.015 \mathrm{~m}^{3}$ ), which determines the power consumption, overall dimensions, and weight of the magnetic system. Since the needed intensity of the magnetic field is comparatively low, it can be produced by both a "warm" solenoid and a cryomagnet. A "warm" solenoid requires a water or oil cooling and, according to calculations, should have an outer diameter of $400 \mathrm{~mm}$, an inner diameter of $205 \mathrm{~mm}$, and a length of $650 \mathrm{~mm}$. The weight of such a solenoid is at least $300 \mathrm{~kg}$ and it needs a dc source of a $5.5 \mathrm{~kW}$ power.

In this particular situation, taking into account the assumed operation conditions of the gyrotron, it was decided to abandon the idea of manufacturing a "warm" solenoid with bulky power sources and cooling systems. Instead of this, a special cryomagnet was manufactured for the gyrotron with an operating frequency of $5 \mathrm{GHz}$. The main parameters of the cryostat are as follows: a height of $1150 \mathrm{~mm}$, a diameter 
of $650 \mathrm{~mm}$, the "warm" bore of $205 \mathrm{~mm}$ in diameter and $760 \mathrm{~mm}$ in length, the capacity of a helium vessel is $71 \mathrm{l}$, the capacity of a nitrogen vessel is $50 \mathrm{l}$, and the solenoid constant is $9.13 \cdot 10^{-3} \mathrm{~T} / \mathrm{A}$.

The material for the energy-output window was boron nitride. The thickness of the window was $15 \mathrm{~mm}$ and its diameter was $100 \mathrm{~mm}$. The calculated losses in the window for an output power of $500 \mathrm{~kW}$ were about $60 \mathrm{~W}$.

The results of the experiments are shown in Fig. 2. Unfortunately, the experiments with this gyrotron stopped in the initial stage, since the T-10 work schedule was modified and the planned experiments, for which this oscillator had been developed, were canceled. Therefore, it was tested only at comparatively low beam currents, which did not exceed $15 \mathrm{~A}$. The obtained output power $P_{\text {out }}$ and efficiency are shown by triangular dots in the graph. For comparison, the same figure shows the calculated curves corresponding to the experimental operation regimes.

Another high-power low-frequency industrial gyrotron was the oscillator developed in 1993. It had an operating frequency of $28 \mathrm{GHz}$ and the maximum output power $0.5 \mathrm{MW}$ for an efficiency of $36 \%$ [8]. These parameters were achieved in the regime of long $(0.1 \mathrm{~s})$ pulses for an accelerating voltage of $70 \mathrm{kV}$ and a beam current of $20 \mathrm{~A}$. The general view and dimensions of the gyrotron are shown in Fig. 3.

The operating mode was the $\mathrm{TE}_{4,2,1}$ mode. The operating wavelength was much shorter compared with the previous gyrotron. It provided an acceptable level of diffraction losses in the axially nonsymmetric output module which made it possible to separate the output electromagnetic radiation and the electron beam. Because of this, a quasi-optical mirror converter of the operating mode into the Gaussian wave beam was used. This beam was output from the tube in the transverse direction.

The converter efficiency reached $90 \%$. The calculated parameters of the magnetron-injection electron gun of the diode type were as follows: the average radius of the emitter was $21.5 \mathrm{~mm}$, the average radius of the electron beam in the cavity was $7 \mathrm{~mm}$, the density of the cathode emission current amounted to $2.5 \mathrm{~A} / \mathrm{cm}^{2}$, the pitch factor, to 1.3 , and the relative spread of electrons over transverse velocities, to $5 \%$ (without allowance for

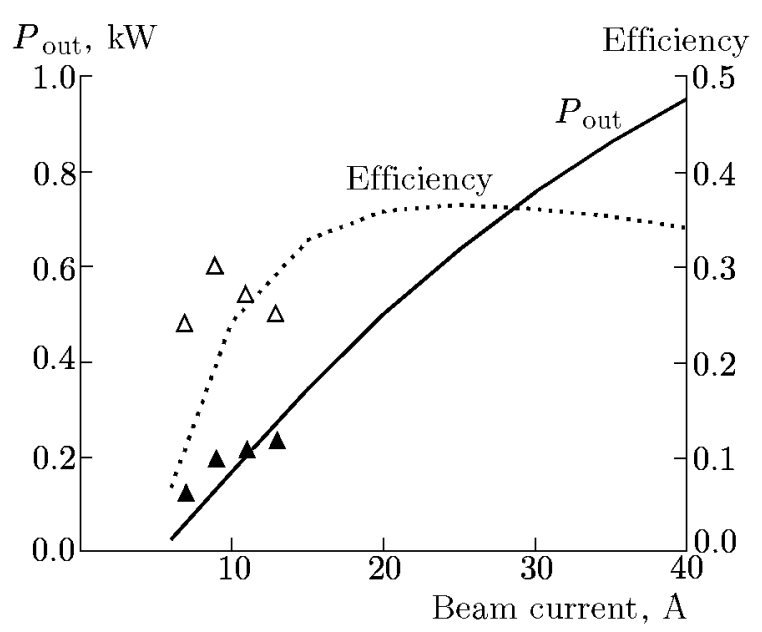

Fig. 2. Results of the experimental study of the gyrotron with an operating frequency of $5 \mathrm{GHz}$, an output power of $0.5 \mathrm{MW}$, a pulse duration of $1 \mathrm{~s}$, and an accelerating voltage of $70 \mathrm{kV}$.

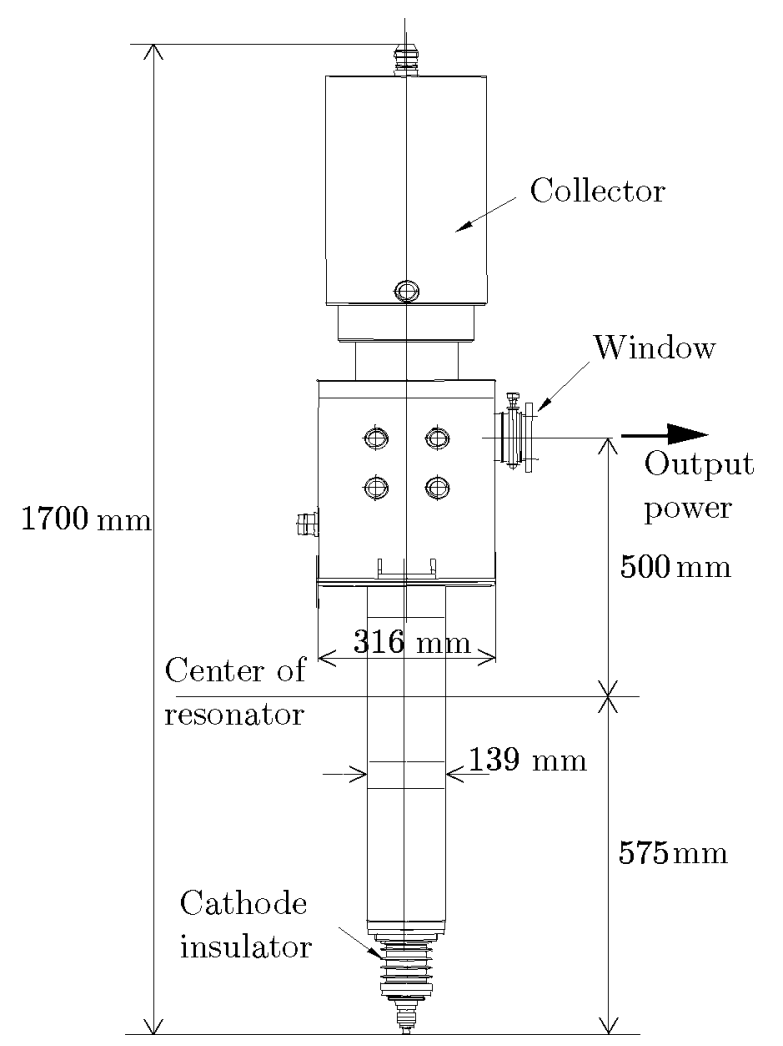

Fig. 3. General view of the gyrotron with an operating frequency of $28 \mathrm{GHz}$, an output power of $0.5 \mathrm{MW}$, and a pulse duration of $0.1 \mathrm{~s}$.

the initial velocities). It should be noted that due to optimization of the geometry of the electron-gun electrodes, the above-mentioned (minimum) value of the relative spread was achieved for an electron-beam operating current equal to $20 \mathrm{~A}$. The results of experimental studies of this gyrotron are shown in Fig. 4. 


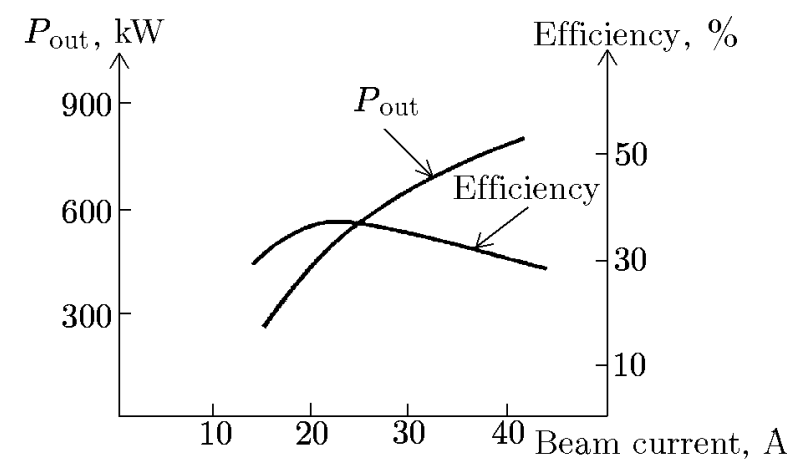

Fig. 4. Results of the experimental studies of the gyrotron shown in Fig. 3.

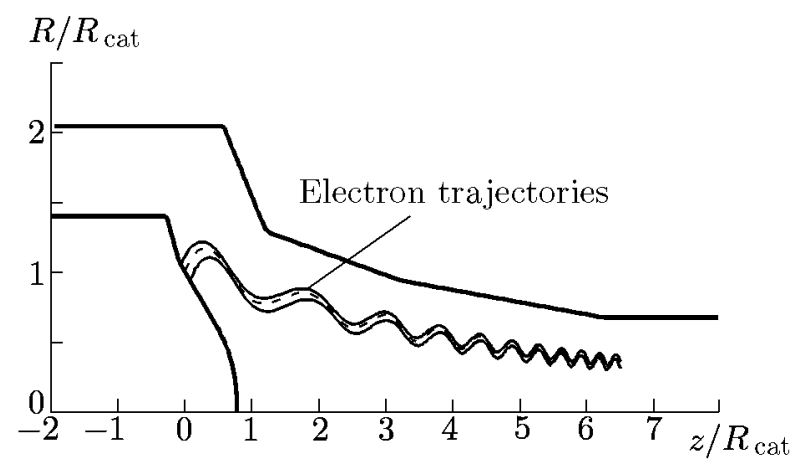

Fig. 5. Configuration of the electrodes of the electron gun and the shape of the electron trajectories for the gyrotron with an operating frequency of $17.5 \mathrm{GHz}$, an output power of $1 \mathrm{MW}$, and a pulse duration $5 \mathrm{~s}$. Here, $z$ is the longitudinal coordinate.

\section{NEW LOW-FREQUENCY GYROTRON PROJECTS}

As was mentioned, devices that currently seem to be promising for development are high-power long-pulse gyrotrons operated in the frequency range 15-30 GHz. In what follows we present calculated parameters of two megawatt long-pulse gyrotrons (pulse duration up to $5 \mathrm{~s}$ ) with operating frequencies 17.5 and $28 \mathrm{GHz}$.

The $\mathrm{TE}_{5,2,1}$ and $\mathrm{TE}_{1,3,1}$ modes were considered operating ones in the designed gyrotron with an operating frequency of $17.5 \mathrm{GHz}$. Finally, the $\mathrm{TE}_{1,3,1}$ mode was chosen. The advantage of this mode is that it becomes a standing wave (in the azimuthal direction) if the waveguide transition from the cavity to the quasi-optical emitter (deflector) is made elliptical. This makes the diffraction losses at the deflector edges significantly lower compared with the rotating $\mathrm{TE}_{5,2,1}$ mode and also decreases the deflector length by two times, and, correspondingly, the size of the entire output electrodynamic system within the limits of the dimensions of technologically mastered modern industrial gyrotrons. The radius of the gyrotron cavity for the operating mode $\mathrm{TE}_{1,3,1}$ is equal to $R_{\text {cav }}=23.8 \mathrm{~mm}$, and the specific thermal load does not exceed $0.3 \mathrm{~kW} / \mathrm{cm}^{2}$.

The configuration of electrodes and trajectories of electrons in the optimized electron-optical system that can be used in such gyrotrons are shown in Fig. 5 on the scale of the average emitter radius $R_{\text {cat }}$. The dependences of the relative spread $\delta v_{\perp}$ of electrons over relative transverse velocities and the relative energy $t_{\perp}$ of the oscillatory motion on the beam current for the model with zero initial velocities $\left(v_{0}=0\right.$; EPOSR [9]) are shown in Fig. $6 a$ (solid lines).

Additional information on the properties of the actual beam can be obtained by considering the model taking the spread of initial velocities into account $\left(v_{0} \neq 0\right.$; EPOS-V [10]). The obtained dependences of $\delta v_{\perp}$ and $t_{\perp}$ on the beam current for the model with $v_{0} \neq 0$ are also presented for comparison in Fig. $6 a$ (dashed lines). Figure $6 b$ shows the functions $F\left(v_{\perp}\right)$ of electron distribution over transverse velocities, normalized to unit magnitude, for different values of the beam current $I$ ( $v_{0}$ is the total electron velocity corresponding to the accelerating voltage $\left.U_{0}\right)$. According to calculations, this gun is capable of forming a helical electron beam with data-sheet accelerating voltage $100 \mathrm{kV}$, current $40 \mathrm{~A}$, pitch factor 1.3, and relative spread over transverse velocities about $20 \%$. The function of distribution in transverse velocities $v_{\perp}$ for different values of the current beam $I$ is shown in Fig. $6 b$.

The next problem which was solved within the framework of this project was the problem of the collector. Figure 7 shows the geometry of the additional collector solenoid and the magnetic-field lines in the collector region in the initial version. The lines filled the collector region uniformly. However, according 

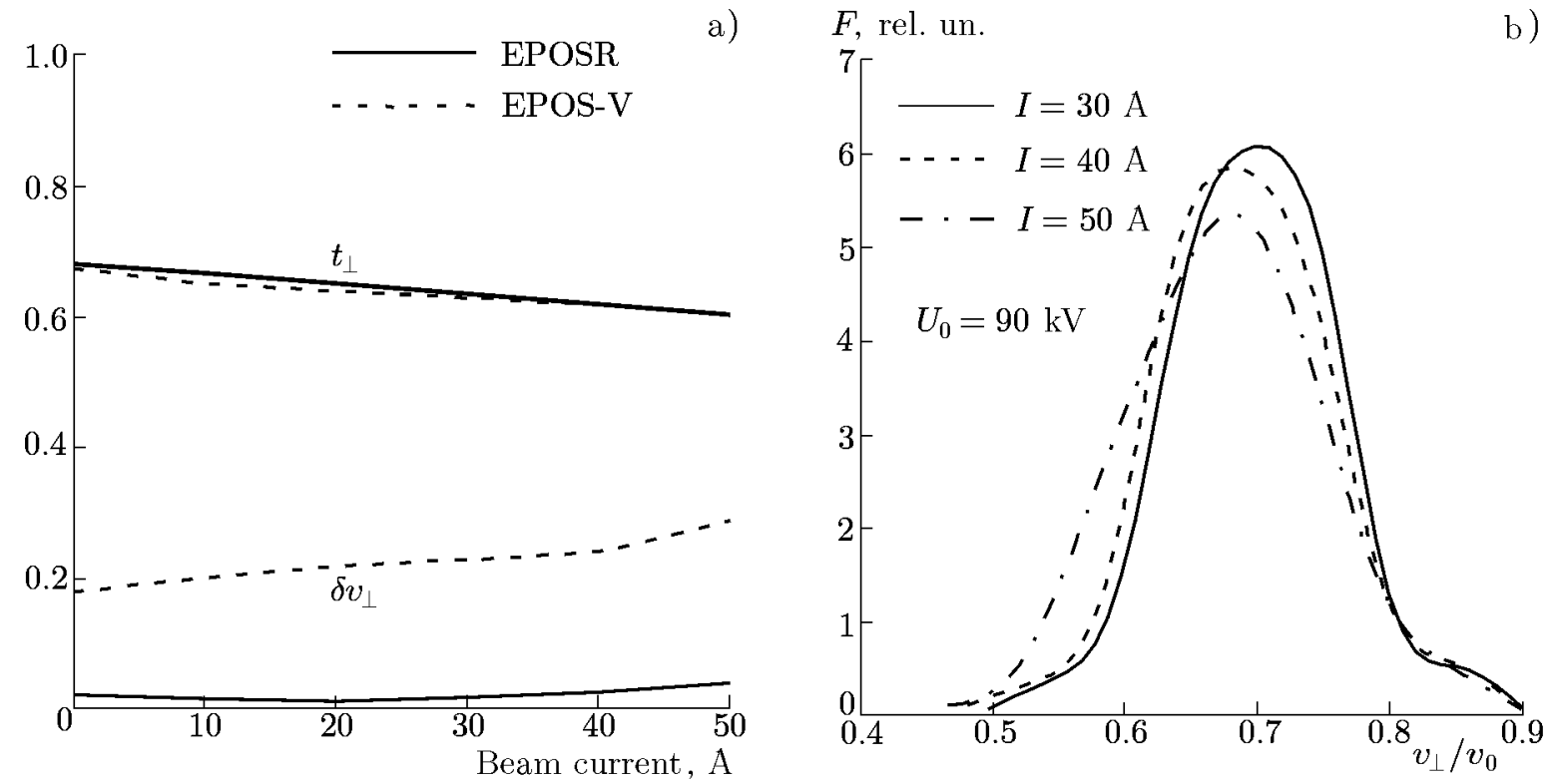

Fig. 6. Calculated parameters of the electron beam in the gyrotron with an operating frequency of $17.5 \mathrm{GHz}$, an output power of $1 \mathrm{MW}$, and a pulse duration of $5 \mathrm{~s}$.

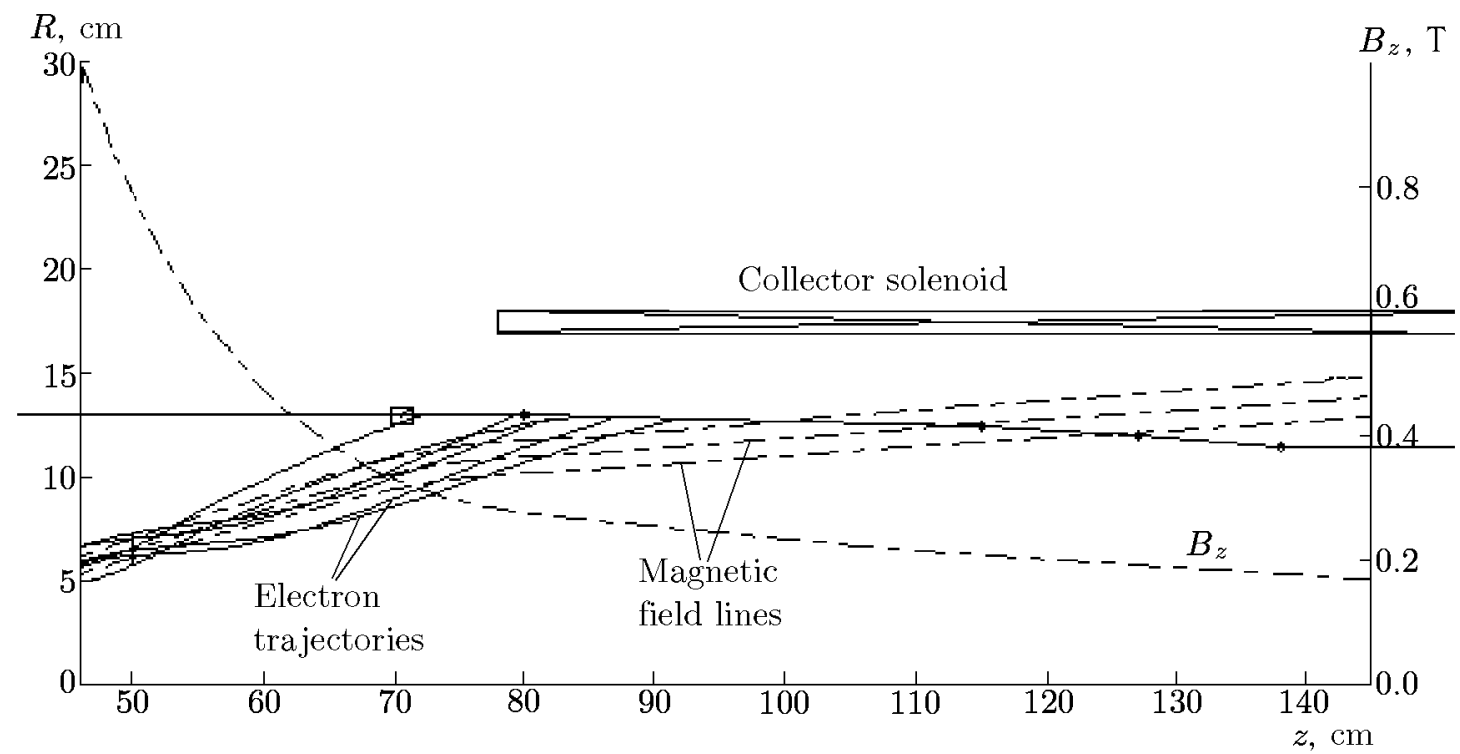

Fig. 7. Magnetic-field lines in the collector region and the electron trajectories with allowance for the space charge in the initial system.

to the trajectory analysis, the distribution of the specific heat load along the collector with average diameter $125 \mathrm{~mm}$ turned out to be significantly nonuniform (Fig. 8). This effect is observed when the cyclotron pitch of the helical trajectory is close to the length of the electron-beam landing, found from the distribution of magnetic-field lines along the collector.

In this connection, another version of the system of collector solenoids has been proposed, which is shown in Fig. 9. It uses additional subtractive-polarity solenoids to introduce perturbations to the magnetic field in front of the collector. As a result, the electron trajectories are mixed up, and the landing of electrons along the collector becomes more uniform.

Figure 10 shows the distribution of the specific thermal load along the collector of $150 \mathrm{~mm}$ in diameter 

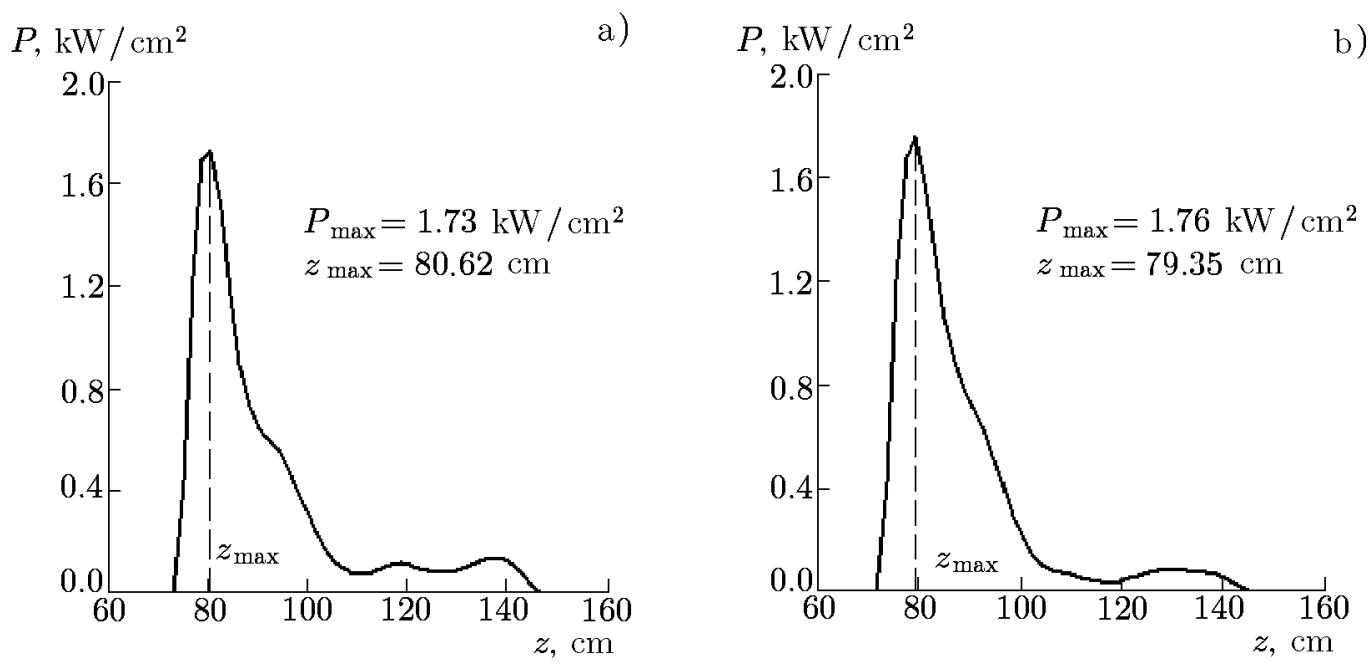

Fig. 8. Result of the trajectory analysis of the electron beam landing on the collector. Distribution of the density of the power scattered along the collector without and with allowance for the space charge (panels a and $b$, respectively).

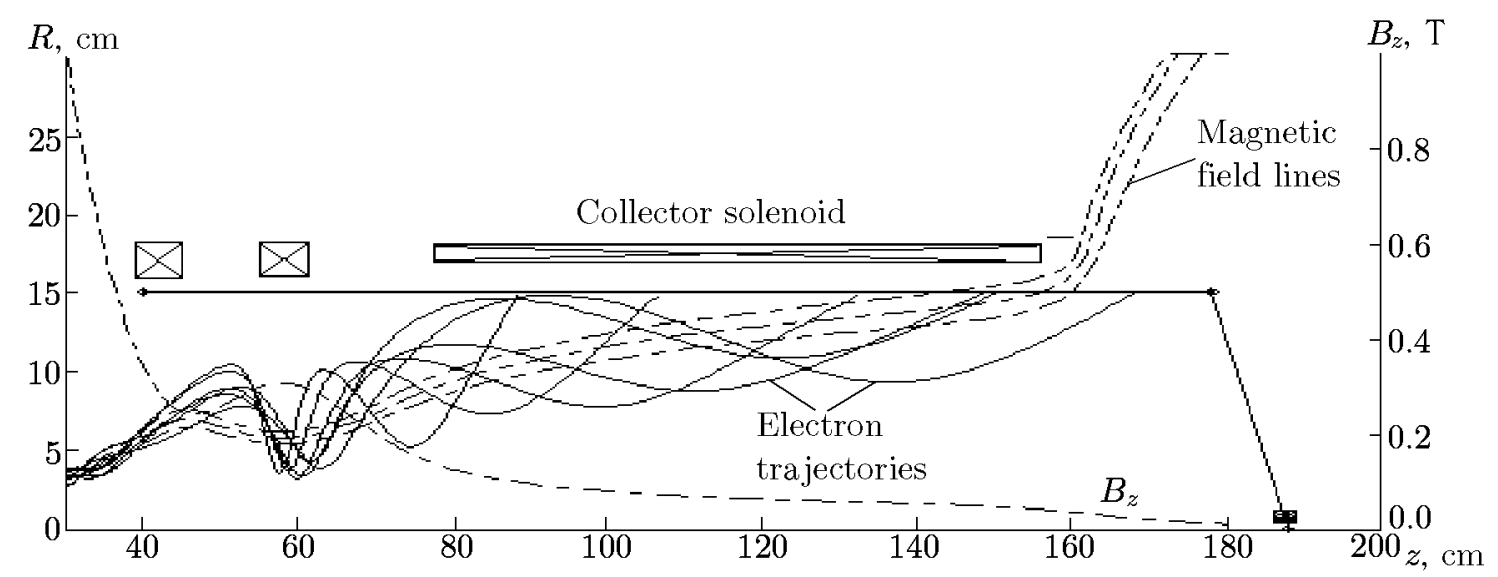

Fig. 9. Magnetic-field lines in the collector region and the electron trajectories in the case of optimized collector solenoids. Notations are the same as in Fig. 8.

in the case of such an optimized system of additional collector solenoids. It is seen that the distribution has become more uniform as compared with the initial version and the peak thermal load not exceeding $0.5 \mathrm{~kW} / \mathrm{cm}^{2}$ is admissible up to the continuous-wave operation regime.

The calculated output parameters of the designed gyrotron with an operating frequency of $17.5 \mathrm{GHz}$ are shown in Fig. 11.

For a shorter-wavelength gyrotron with the operating frequency $28 \mathrm{GHz}$, output power $1 \mathrm{MW}$, and pulse duration $5 \mathrm{~s}$, the $\mathrm{TE}_{6,2,1}$ mode was chosen to be the operating one. At a frequency of $28 \mathrm{GHz}$, the diffraction losses at the edges of the waveguide cut are not too strong, and a typical design scheme of a gyrotron output module based on the rotating mode is quite acceptable. This is confirmed by the experience of operating the above-described gyrotron with a frequency of $28 \mathrm{GHz}$ and an output power of $0.5 \mathrm{MW}$ at the operating mode $\mathrm{TE}_{4,2,1}$. In terms of the thermal load in the cavity, the lower modes, such as the $\mathrm{TE}_{5,2,1}$ mode are also admissible, but the $\mathrm{TE}_{6,2,1}$ mode was eventually chosen (the calculated specific thermal load does not exceed $0.4 \mathrm{~kW} / \mathrm{cm}^{2}$ ) since it is most convenient for transformation into the Gaussian wave beam by using a high-efficiency adiabatic converter [11]. According to the preliminary drawings, the designed gyrotron will be similar in external appearance to the industrial model shown in Fig. 3, but will have a 


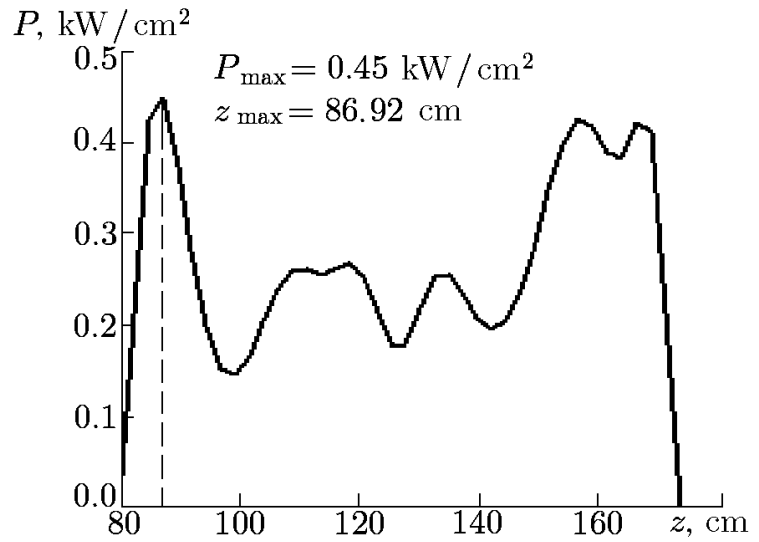

Fig. 10. Distribution of the specific thermal load along the collector in an optimized system of solenoids.

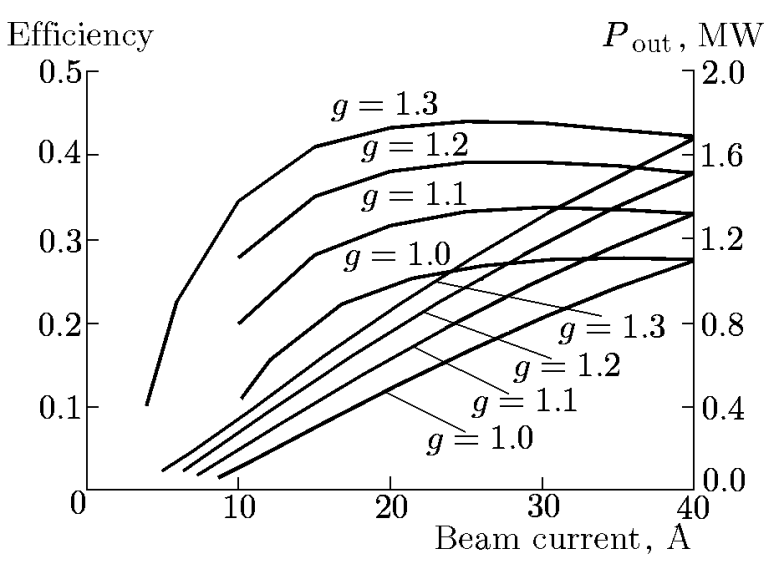

Fig. 11. Calculated output parameters of a gyrotron with an operating frequency of $17.5 \mathrm{GHz}$, an output power of $1 \mathrm{MW}$, and a pulse duration of $5 \mathrm{~s}$ for the accelerating voltage $U_{0}=100 \mathrm{kV}$, the operating mode $\mathrm{TE}_{1,3,1}$, and the cavity Q-factor $Q=494$.

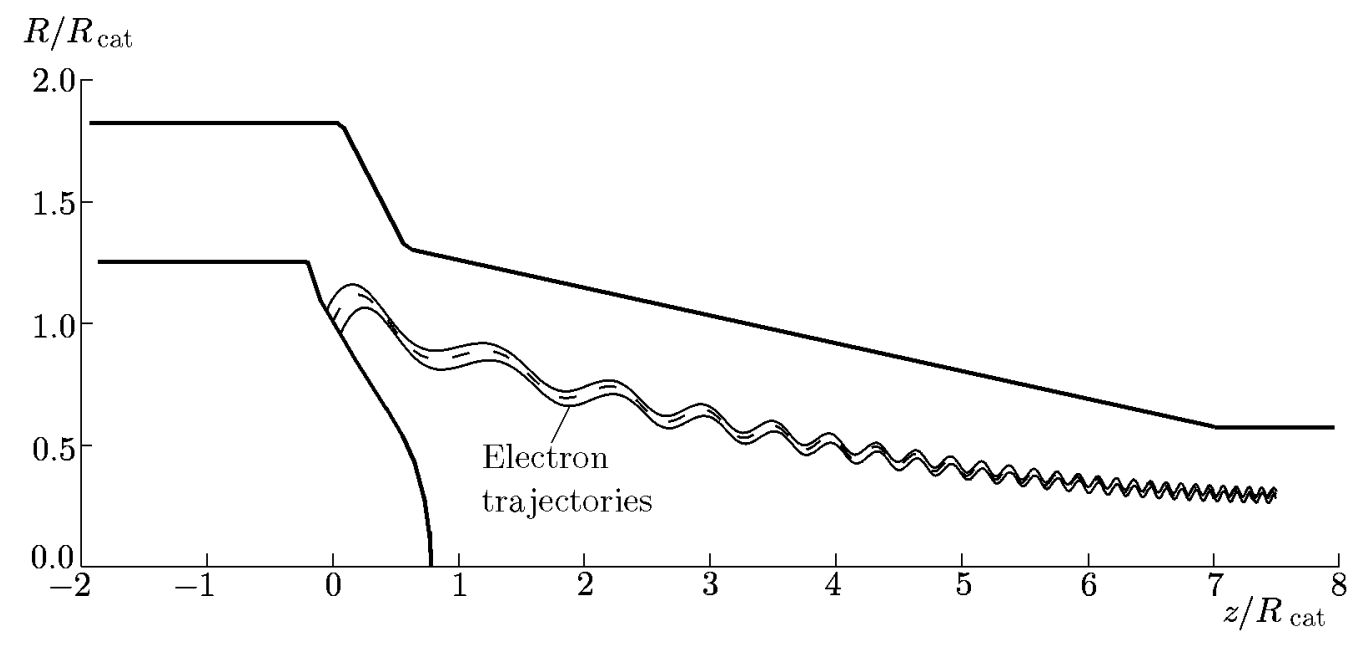

Fig. 12. Configuration of the electrodes of the electron gun and the form of the electron trajectories for a gyrotron with an operating frequency of $28 \mathrm{GHz}$, an output power of $1 \mathrm{MW}$, and a pulse duration of $5 \mathrm{~s}$.

more voluminous collector capable of operating for a pulse duration of $5 \mathrm{~s}$ and an electron-beam power of up to $4 \mathrm{MW}$. At this frequency, the collector problem is not as acute and can be solved by using the static or dynamic distribution of the electron beam over the collector, which is usually applied in high-power gyrotrons of the millimeter-wave range.

The configuration of the electron gun calculated for such a gyrotron is shown in Fig. 12. The main parameters of the formed electron beam are presented in Fig. 13. The calculated output characteristics of the gyrotron as functions of the beam current are shown in Fig. 14.

\section{CONCLUSIONS}

The paper presents the results of the studies aimed at creation of high-power, long-pulse, lowfrequency gyrotrons in Russia. A number of specific problems which should be solved for the creation of 

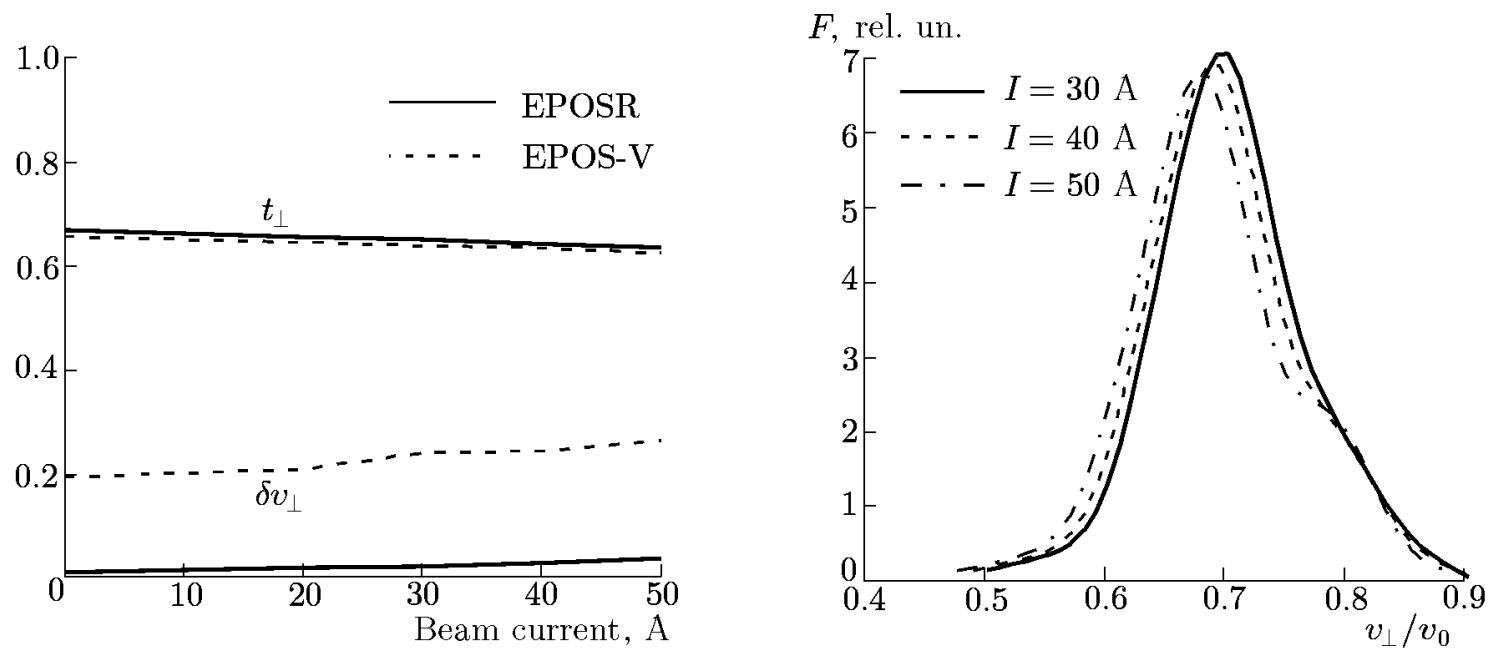

Fig. 13. Main calculated parameters of the electron beam of a gyrotron with an operating frequency of $28 \mathrm{GHz}$, an output power of $1 \mathrm{MW}$, and a pulse duration of $5 \mathrm{~s}$ for the accelerating voltage $U_{0}=90 \mathrm{kV}$ and the cathode radius $R_{\text {cat }}=38 \mathrm{~mm}$.

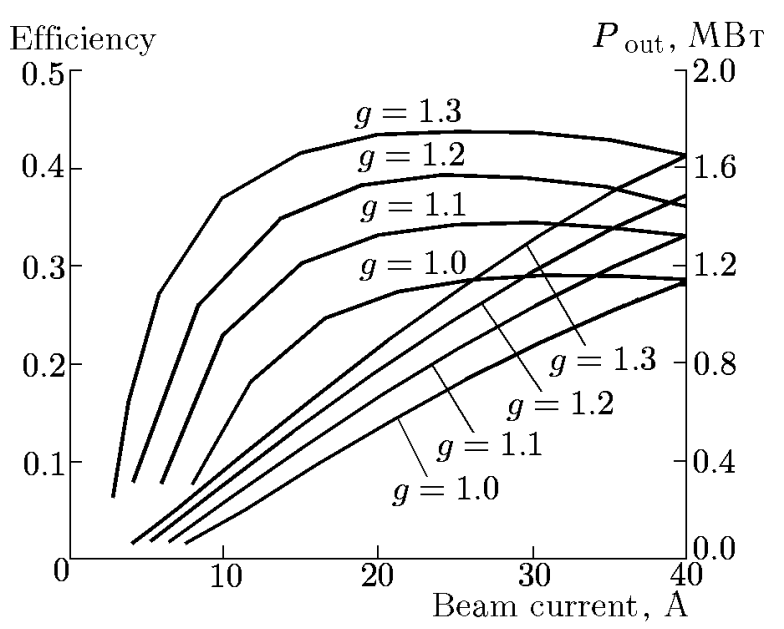

Fig. 14. Calculated output parameters of a gyrotron with the operating frequency $28 \mathrm{GHz}$, output power $1 \mathrm{MW}$, and pulse duration $5 \mathrm{~s}$ for the accelerating voltage $U_{0}=100 \mathrm{kV}$, the operating mode $\mathrm{TE}_{6,2,1}$, and the cavity $\mathrm{Q}$-factor $Q=683$. such gyrotrons were delineated and discussed. Specific features of the design were shown, and operating parameters were presented for industrial gyrotrons with an output power of $0.5 \mathrm{MW}$ and operating frequencies 5 and $28 \mathrm{GHz}$. Design versions of gyrotrons with operating frequencies 17.5 and $28 \mathrm{GHz}$ and an output power of up to $1 \mathrm{MW}$ for a pulse duration of up to $5 \mathrm{~s}$ were discussed.

This work was supported by the Russian Foundation for Basic Research (projects Nos. 03-02-17560, 0502-16015, and 05-02-08024-ofi-a), the INTAS (project No. 03-51-3861), and the Council of the President of the Russian Federation for the State Support of the Leading Scientific Schools (project No. NSh1640.2003.2).

The authors are grateful to S. S. Davydenko for valuable advice and help with the preparation of this paper.

\section{REFERENCES}

1. V. V. Alikaev, G. G. Denisov, V.E. Zapevalov, et al., in: High-Frequency Vacuum Electronics, Collection of Review Papers [in Russian], Inst. Appl. Phys., Nizhny Novgorod (2002), p. 71.

2. V.E. Zapevalov, V.I. Belousov, A. A. Bogdashov, et al., in: 28th Int. Conf. on Infrared and Millimeter Waves, Otsu, Japan (2003), p. 165.

3. Yu. Bykov, A. Eremeev, M. Glyavin, et al., IEEE Trans. Plasma Sci., 32, No. 1, 162 (2004).

4. V.F. Shevchenko, Y. Baranov, M. O. Brien, et al., in: Proc. 13th Joint Workshop on Electron Cyclotron Emission and Electron Cyclotron Heating, Nizhny Novgorod, Russia, May 17-20, 2004, p. 162. 
5. V.F.Shevchenko, Y. Baranov, A. N. Saveliev, et al., in: Proc. 13th Joint Workshop on Electron Cyclotron Emission and Electron Cyclotron Heating, Nizhny Novgorod, Russia, May 17-20, 2004, p. 255.

6. V.E.Zapevalov, Yu. K. Kalynov, V.I. Khizhnjak et al., in: Proc. 13th Joint Workshop on Electron Cyclotron Emission and Electron Cyclotron Heating, Nizhny Novgorod, Russia, May 17-20, 2004, p. 403.

7. A. L. Gol'denberg and M. I. Petelin, Radiophys. Quantum Electron., 16, No. 1, 106 (1973).

8. S. D. Bogdanov, V.I. Kurbatov, S. A. Malygin, et al., in: Strong Microwaves in Plasmas, Inst. Appl. Phys., Nizhny Novgorod (1993), Vol. 2, p. 834.

9. V.K. Lygin, V.N. Manuilov, and Sh.E.Tsimring, Élektron. Tekhn., Ser. Élektron. SVCh, No.7, 36 (1987).

10. V. K. Lygin, Int. J. Infrared Millimeter Waves, 16, No. 2, 363 (1995).

11. G. G. Denisov, A. N. Kuftin, V. I. Malygin, et al., Int. J. Electron., 72, Nos. 5-6, 1079 (1992). 